\title{
Wiener-Hopf determinants with Fisher-Hartwig symbols
}

\author{
Estelle L. Basor \\ Department of Mathematics \\ California Polytechnic State University \\ San Luis Obispo, CA 93407, USA
}

\author{
Harold Widom \\ Department of Mathematics \\ University of California \\ Santa Cruz, CA 95064, USA
}

\section{Introduction.}

The strong Szegö limit theorem states that if the symbol $\varphi$ defined on the unit circle has a sufficiently well-behaved logarithm then the determinant of the Toeplitz matrix

$$
T_{n}(\varphi)=\left(\varphi_{j-k}\right)_{j, k=0, \cdots, n-1}
$$

has the asymptotic behavior

$$
\operatorname{det} T_{n}(\varphi) \sim G(\varphi)^{n} E(\varphi) \text { as } n \rightarrow \infty
$$

where

$$
G(\varphi)=e^{(\log \varphi)_{0}}, \quad E(\varphi)=\exp \left(\sum_{k=1}^{\infty} k(\log \varphi)_{k}(\log \varphi)_{-k}\right) .
$$

Here subscripts denote Fourier coefficients.

Fisher and Hartwig [12 introduced a family of symbols with singularities and conjectured the form of the asymptotics for these. If

$$
\varphi_{\alpha, \beta}\left(e^{i \theta}\right)=(2-2 \cos \theta)^{(\alpha+\beta) / 2} e^{i(\theta-\pi)(\alpha-\beta) / 2}, \quad 0<\theta<2 \pi
$$

(this symbol is said to have a pure Fisher-Hartwig singularity), then their symbols had the form

$$
\psi(z)=\varphi(z) \prod_{j=1}^{N} \varphi_{\alpha_{j}, \beta_{j}}\left(z / z_{j}\right)
$$

where $\varphi$ satisfies the assumption of Szegö's theorem and $z_{1}, \cdots, z_{N}$ are distinct points on the unit circle. They conjectured that for some range of the parameters the asymptotics had the form

$$
\operatorname{det} T_{n}(\psi) \sim E\left(\varphi, \alpha_{j}, \beta_{j}, z_{j}\right) n^{-\sum \alpha_{j} \beta_{j}}
$$

where $E\left(\varphi, \alpha_{j}, \beta_{j}, z_{j}\right)$ is a constant (whose value they did not conjecture). Due to the work of many mathematicians the conjecture has now been proved, and the constant $E\left(\varphi, \alpha_{j}, \beta_{j}, z_{j}\right)$ determined, in great generality. The basic condition is that $\left|\Re\left(\alpha_{j} \pm \beta_{j}\right)\right|<1$. In early work the case of several singularities was tackled directly and the proofs were quite involved. But later it was discovered [1, 3, 6] that one could use localization techniques which 
made it possible to prove general results if one knew the asymptotics for the symbols $\varphi_{\alpha, \beta}$ with pure singularity. Luckily the Toeplitz determinants in these cases could be evaluated explicitly and their asymptotics were then a straightforward matter [7]. (For a detailed history, and a proof of the general result, see [8]. Also, results for symbols with one singularity are contained in [10], and a status report for a generalized conjecture can be found in [11.)

For the Wiener-Hopf analogue the symbol $\sigma(\xi)$ is defined on the real line and equals 1 at $\pm \infty$. The finite Wiener-Hopf operator $W_{R}(\sigma)$ acts on $L^{2}(0, R)$ and is equal to the identity plus the operator with kernel

$$
\int_{-\infty}^{\infty}(\sigma(\xi)-1) e^{i(x-y) \xi} d \xi
$$

Here there is also a "Szegö theorem" for nicely-behaved symbols:

$$
\operatorname{det} W_{R}(\sigma) \sim G(\sigma)^{R} E(\sigma) \text { as } R \rightarrow \infty .
$$

Now

$$
G(\sigma)=e^{\tau(0)}, \quad E(\sigma)=\exp \left(\int_{0}^{\infty} x \tau(x) \tau(-x) d x\right)
$$

where $\tau$ is the Fourier transform of $\log \sigma$,

$$
\tau(x)=\frac{1}{2 \pi} \int_{-\infty}^{\infty} \log \sigma(\xi) e^{-i x \xi} d \xi .
$$

A Wiener-Hopf symbol with pure Fisher-Hartwig singularity could be defined by

$$
\sigma_{\alpha, \beta}(\xi)=\left(\frac{\xi-0 i}{\xi-i}\right)^{\alpha}\left(\frac{\xi+0 i}{\xi+i}\right)^{\beta}
$$

(We specify the arguments of $\xi \pm 0 i$ and $\xi \pm i$ to be zero or close to it when $\xi$ is large and positive.) This has the behavior

$$
\sigma_{\alpha, \beta}(\xi) \sim|\xi|^{\alpha+\beta} e^{\frac{1}{2} i \pi(\alpha-\beta) \operatorname{sgn} \xi} \quad \text { as } \xi \rightarrow 0
$$

One would like to prove an analogue of the Fisher-Hartwig conjecture for symbols which have a finite number of singularities of the above type. It seems reasonable to try to do what was done in the Toeplitz case, that is, devise the proper localization techniques and then try to evaluate the determinants in the case of the pure singularity. However, while it is likely that quite general localization techniques can be developed (and, in fact, have been in certain cases) there has never been an single example where the finite Wiener-Hopf determinant for a singular symbol could be evaluated explicitly. So far results of any generality are known only for symbols with jump discontinuities, when all $\alpha_{j}+\beta_{j}=0$ [4, 9]. These were obtained by (two different kinds of) discretization which led to Toeplitz problems.

In this paper we shall find the Wiener-Hopf asymptotics for the pure symbols $\sigma_{\alpha, \beta}$. A minor complication encountered for these symbols is that except when $\alpha=\beta$ the function $\sigma_{\alpha, \beta}-1$ is not in $L^{1}$ but in $L^{2}$. Hence even to consider an analogue of the formula for Toeplitz determinants with pure Fisher-Hartwig singularities, we must use the regularized determinants $\operatorname{det}_{2} W_{R}(\sigma)$. 
We determine the asymptotics for the symbols $\sigma_{\alpha, \beta}$ under the condition $|\Re(\alpha \pm \beta)|<1$. This is done, not by evaluating the regularized determinant explicitly, but by showing that after normalization it is asymptotically equal to a regularized Toeplitz determinant.

Theorem. If $|\Re(\alpha \pm \beta)|<1$ then

$$
\operatorname{det}_{2} W_{R}\left(\sigma_{\alpha, \beta}\right) / G_{2}\left(\sigma_{\alpha, \beta}\right)^{R} \sim \operatorname{det}_{2} T_{n}\left(\varphi_{\alpha, \beta}\right) / G_{2}\left(\varphi_{\alpha, \beta}\right)^{n}
$$

when $R \sim 2 n \rightarrow \infty$, and where

$$
G_{2}(\sigma)=\exp \left(\frac{1}{2 \pi} \int_{-\infty}^{\infty}(\log \sigma(\zeta)-\sigma(\zeta)+1) d \zeta\right)
$$

and

$$
G_{2}(\varphi)=\exp \left((\log \varphi)_{0}-\varphi_{0}+1\right) .
$$

This will be proved by finding exact formulas for the Toeplitz and Wiener-Hopf determinants and regularized determinants and then showing the above quotients are asymptotically equal when $R \sim 2 n$. These formulas are expressed in terms of Fredholm determinants of operators acting on $L^{2}(0,1)$ and are obtained by using an identity of Borodin and Okounkov [5] for Toeplitz determinants with regular symbol and its Wiener-Hopf analogue [2]. What we do is simply stated: in both cases we introduce a parameter to regularize the symbol, apply the identity, and then take the limit.

We remark that in the Toeplitz case, computing a regularized determinant from an ordinary determinant is easy and it causes no problem to go back and forth between the two in computations. The same is true for the finite Wiener-Hopf operators when $\sigma_{\alpha, \beta}-1$ is in both $L^{2}$ and $L^{1}$. This holds exactly when $\beta=\alpha$, and the following corollary will follow from the theorem.

Corollary. If $|\Re(\alpha)|<1 / 2$ then

$$
\operatorname{det} W_{R}\left(\sigma_{\alpha, \alpha}\right) \sim \operatorname{det} T_{n}\left(\varphi_{\alpha, \alpha}\right)
$$

when $R \sim 2 n \rightarrow \infty$.

\section{The case of $\beta=\alpha$.}

We do this case first since the general case has extra complications, but the main points are the same and will not have to be repeated.

To state the Borodin-Okounkov identity, let $\varphi$ be a symbol with regular logarithm and let $\varphi(z)=\varphi^{-}(z) \varphi^{+}(z)$ be its Wiener-Hopf factorization, so that $\varphi^{+}$extends to a nonzero analytic function inside the unit circle and $\varphi^{-}$outside. Let $K_{n}$ be the operator on $\ell^{2}(\{0,1, \cdots\})$ with matrix entries

$$
K_{n}(i, j)=\sum_{k=0}^{\infty}\left(\varphi^{-} / \varphi^{+}\right)_{n+i+k+1}\left(\varphi^{+} / \varphi^{-}\right)_{-n-j-k-1} .
$$

The identity is

$$
\operatorname{det} T_{n}(\varphi)=G(\varphi)^{n} E(\varphi) \operatorname{det}\left(I-K_{n}\right)
$$


where $G(\varphi)$ and $E(\varphi)$ are the constants appearing in (1). For the regularized determinant we have

$$
\operatorname{det}_{2} T_{n}(\varphi)=G_{2}(\varphi)^{n} E(\varphi) \operatorname{det}\left(I-K_{n}\right) .
$$

This follows immediately from the first identity since

$$
\operatorname{det}_{2} A=\operatorname{det} A e^{-\operatorname{tr}(A-I)}
$$

whenever $A-I$ is trace class. Notice also that we encounter the same expression in computing either

$$
\operatorname{det} T_{n}(\varphi) / G(\varphi)^{n}
$$

or

$$
\operatorname{det}_{2} T_{n}(\varphi) / G_{2}(\varphi)^{n}
$$

so the crucial computation is to find $E(\varphi) \operatorname{det}\left(I-K_{n}\right)$. This will be true when we do the Wiener-Hopf analogue as well.

If we introduce a parameter $r<1$ the symbol $\varphi_{\alpha, \alpha}(z)=(1-z)^{\alpha}\left(1-z^{-1}\right)^{\alpha}$ becomes $\varphi_{r}(z)=(1-r z)^{\alpha}\left(1-r z^{-1}\right)^{\alpha}$, a regular symbol which is in $L^{1}$ and for which both (3) and (国) hold. The limit as $r$ tends to one of $G_{2}\left(\varphi_{r}\right)$ is easily seen to be $G_{2}\left(\varphi_{\alpha, \alpha}\right)$. The constant $E\left(\varphi_{r}\right)$ equals $\left(1-r^{2}\right)^{-\alpha^{2}}$. The operator $K_{n}$ is the product of two Hankel operators on $\ell^{2}(\{0,1, \cdots\})$ which in this case are the same and have $i, j$ entry

$$
\frac{1}{2 \pi i} \int_{|z|=1}\left(\frac{1-r z}{1-r z^{-1}}\right)^{\alpha} z^{n+i+j} d z=\frac{\sin \pi \alpha}{\pi} \int_{0}^{r}\left(\frac{1-r x}{r-x}\right)^{\alpha} x^{n+i+j+\alpha} d x .
$$

If the operator is denoted by $H$ then $\operatorname{det}\left(I-K_{n}\right)=\operatorname{det}\left(I-H^{2}\right)$, and so we are interested in $\operatorname{det}(I \pm H)$. We consider at first only $\operatorname{det}(I-H)$, the Fredholm determinant of $H$.

If $\Re \alpha<1 / 2$ we can write $H=U V$, where $U: L^{2}(0, r) \rightarrow \ell^{2}(\{0,1, \cdots\})$ has kernel $U(i, x)=x^{i}$ and $V: \ell^{2}(\{0,1, \cdots\}) \rightarrow L^{2}(0, r)$ has kernel

$$
V(x, i)=\frac{\sin \pi \alpha}{\pi}\left(\frac{1-r x}{r-x}\right)^{\alpha} x^{n+i+\alpha} .
$$

These are both Hilbert-Schmidt (in fact trace class) and $\operatorname{det}(I-U V)=\operatorname{det}(I-V U)$. (See, for example, Chap. 4 of [13].) It follows that $H$ has the same Fredholm determinant as the kernel

$$
\frac{\sin \pi \alpha}{\pi}\left(\frac{1-r x}{r-x}\right)^{\alpha} x^{n+\alpha} \frac{1}{1-x y}
$$

on $L^{2}(0, r)$. If we set $r=(1-\varepsilon) /(1+\varepsilon)$ and make the substitutions

$$
x \rightarrow \frac{1-x}{1+x}, \quad y \rightarrow \frac{1-y}{1+y},
$$

this becomes the kernel

$$
\frac{\sin \pi \alpha}{\pi}\left(\frac{x+\varepsilon}{x-\varepsilon}\right)^{\alpha}\left(\frac{1-x}{1+x}\right)^{n+\alpha} \frac{1}{x+y}
$$


on $L^{2}(\varepsilon, 1)$. (By this we mean that the kernels represent unitarily equivalent operators and so have the same Fredholm determinant.) We shall determine the limit of its Fredholm determinant as $\varepsilon \rightarrow 0$.

Denote by $A$ the operator with the above kernel and by $A_{0}$ the operator with kernel

$$
A_{0}(x, y)=\frac{\sin \pi \alpha}{\pi} \frac{1}{x+y}
$$

Let $P$ be multiplication by $\chi_{[\sqrt{\varepsilon}, 1]}$ and $Q$ multiplication by $\chi_{[\varepsilon, \sqrt{\varepsilon}]}$. Although our operators act on $L^{2}(\varepsilon, 1)$ they can be thought of in the obvious way of acting on $L^{2}(0,1)$. For example the kernel of $A_{0}$ can be replaced by $\chi_{[\varepsilon, 1]}(x) A_{0}(x, y) \chi_{[\varepsilon, 1]}(y)$.

We shall show that $P\left(A-A_{0}\right)$ converges in trace norm as $\varepsilon \rightarrow 0$. For this, and later use, we give an estimate for the trace norm of certain kernels.

Lemma 1. The trace norm of a kernel $f(x) g(y) /(x+y)$ on $L^{2}(J)$, where $J \subset(0, \infty)$, is at most a constant depending on $a$ times the square root of

$$
\int_{J}|f(x)|^{2} \frac{d x}{x^{1+a}} d x \int_{J}|g(x)|^{2} \frac{d x}{x^{1-a}} d x
$$

Here $a$ belongs to $(-1,1)$ but is otherwise arbitrary.

Proof. If we write the kernel as

$$
\int_{0}^{\infty} f(x) s^{a / 2} e^{-s x} e^{-s y} s^{-a / 2} g(y) d s
$$

we see that it is the (operator) product of two kernels acting between $L^{2}(J)$ and $L^{2}(0, \infty)$. The square of the Hilbert-Schmidt norm of the first equals

$$
\int_{J} \int_{0}^{\infty}|f(x)|^{2} s^{a} e^{-2 s x} d s d x
$$

which is a constant depending on $a$ times $\int_{J}|f(x)|^{2} x^{-1-a} d x$. The second is analogous.

Lemma 2. The operator $P\left(A-A_{0}\right)$ converges in trace norm to the operator on $L^{2}(0,1)$ with kernel

$$
\frac{\sin \pi \alpha}{\pi}\left[\left(\frac{1-x}{1+x}\right)^{n+\alpha}-1\right] \frac{1}{x+y} .
$$

Proof. The kernel of the operator is the sine factor times

$$
\chi_{[\sqrt{\varepsilon}, 1]}(x)\left[\left(\frac{x+\varepsilon}{x-\varepsilon}\right)^{\alpha}\left(\frac{1-x}{1+x}\right)^{n+\alpha}-1\right] \frac{1}{x+y}
$$

on $L^{2}(\varepsilon, 1)$. If we replace the first $x$-factor by 1 then the error is of the form

$$
\chi_{[\sqrt{\varepsilon}, 1]}(x) \varepsilon_{1}(x)\left(\frac{1-x}{1+x}\right)^{n+\alpha} \frac{1}{x+y}
$$


where $\varepsilon_{1}=O(\sqrt{\varepsilon})$. If we apply Lemma 1 with small positive $a$ and find that the operator has trace norm $O\left(\varepsilon^{1 / 2-\delta}\right)$ for any $\delta>0$. After this replacement we are left with

$$
\chi_{[\sqrt{\varepsilon}, 1]}(x)\left[\left(\frac{1-x}{1+x}\right)^{n+\alpha}-1\right] \frac{1}{x+y},
$$

so it suffices to show that

$$
\left[\left(\frac{1-x}{1+x}\right)^{n+\alpha}-1\right] \frac{1}{x+y}
$$

is trace class on $L^{2}(0,1)$. This also follows from Lemma 1.

Lemma 3. If $\alpha$ is sufficiently small we have as $\varepsilon \rightarrow 0$

$$
\begin{aligned}
\operatorname{det}\left(I-K_{n}\right) & \sim \operatorname{det}\left(I-A_{0}^{2}\right) \operatorname{det}\left(I-\left(I-A_{0}\right)^{-1} P\left(A-A_{0}\right)\right) \operatorname{det}\left(I+\left(I+A_{0}\right)^{-1} P\left(A-A_{0}\right)\right) \\
& \times \operatorname{det}\left(I-\left(\left(I-A_{0}\right)^{-1} Q\left(A-A_{0}\right)\right) \operatorname{det}\left(I+\left(\left(I+A_{0}\right)^{-1} Q\left(A-A_{0}\right)\right) .\right.\right.
\end{aligned}
$$

Proof. Recall that $\operatorname{det}\left(I-K_{n}\right)=\operatorname{det}\left(I-H^{2}\right)$ and that $\operatorname{det}(I \pm H)=\operatorname{det}(I \pm A)$. If $\alpha$ is sufficiently small then $I-A_{0}$ is invertible for all $\varepsilon$ and

$$
\operatorname{det}(I-A)=\operatorname{det}\left(I-A_{0}\right) \operatorname{det}\left(I-\left(I-A_{0}\right)^{-1}\left[P\left(A-A_{0}\right)+Q\left(A-A_{0}\right)\right]\right) .
$$

The operator in the second determinant can be written

$$
\left(I-\left(I-A_{0}\right)^{-1} Q\left(A-A_{0}\right)\right)\left(I-\left(I-A_{0}\right)^{-1} P\left(A-A_{0}\right)\right)-\left(I-A_{0}\right)^{-1} Q\left(A-A_{0}\right)\left(I-A_{0}\right)^{-1} P\left(A-A_{0}\right) .
$$

Now the operators $I-A_{0}$ are uniformly invertible (their inverses have bounded norms as $\varepsilon \rightarrow 0)$ if $\alpha$ is small enough, because of the sine factor in $A_{0}$. The same is true of the first product above, because of the sine factor in $A-A_{0}$. It follows that (6) will be established of we can show that the last term above is $o_{1}(1)$, i.e, its trace norm is $o(1)$. (And if the analogous statement holds for $I+A$, which it will.) We know that $P\left(A-A_{0}\right)$ converges in trace norm. It follows from the uniform invertibility of $\left(I-A_{0}\right)^{-1}$ that it converges strongly as $\varepsilon \rightarrow 0$ to the corresponding operator on $L^{2}(0,1)$, where $A_{0}$ does not have the $\chi_{[\varepsilon, 1]}$ factors. The same is clearly true of $A-A_{0}$. Hence, since $Q$ converges strongly to 0 , the same is true of $Q\left(A-A_{0}\right)\left(I-A_{0}\right)^{-1}$ and this together with the trace norm convergence of $P\left(A-A_{0}\right)$ implies that the last term above is $o_{1}(1)$.

We now have the ingredients necessary to derive our formula for $\operatorname{det}_{2} T_{n}\left(\varphi_{\alpha, \alpha}\right) / G_{2}\left(\varphi_{\alpha, \alpha}\right)^{n}$.

Lemma 4. Assume $|\Re \alpha|<1 / 2$. Let $A_{0}$ be the operator with kernel

$$
A_{0}(x, y)=\frac{\sin \pi \alpha}{\pi} \frac{1}{x+y}
$$

$A_{1}$ the operator with kernel

$$
A_{1}(x, y)=\frac{\sin \pi \alpha}{\pi}\left[\left(\frac{1-x}{1+x}\right)^{n+\alpha}-1\right] \frac{1}{x+y},
$$


and $A_{2}$ the operator with kernel

$$
A_{2}(x, y)=\frac{\sin \pi \alpha}{\pi}\left[\left(\frac{1+x}{1-x}\right)^{\alpha}-1\right] \frac{1}{x+y} .
$$

All act on $L^{2}(0,1)$. Then

$$
\operatorname{det}_{2} T_{n}\left(\varphi_{\alpha, \alpha}\right) / G_{2}\left(\varphi_{\alpha, \alpha}\right)^{n}=4^{-\alpha^{2}} E(1-\sin \pi \alpha \operatorname{sech} \pi \xi) E(1+\sin \pi \alpha \operatorname{sech} \pi \xi)
$$

$\times \operatorname{det}\left(I-\left(I-A_{0}\right)^{-1} A_{1}\right) \operatorname{det}\left(I+\left(I+A_{0}\right)^{-1} A_{1}\right) \operatorname{det}\left(I-\left(I-A_{0}\right)^{-1} A_{2}\right) \operatorname{det}\left(I+\left(I+A_{0}\right)^{-1} A_{2}\right)$, where the $E$ factors are those of (2).

Proof. We compute the asymptotics of the right side of (16) as $\varepsilon \rightarrow 0(r \rightarrow 1)$. If we make the substitutions $x \rightarrow e^{-x}, y \rightarrow e^{-y}$ then $A_{0}$ becomes the operator on $L^{2}\left(0, \log \varepsilon^{-1}\right)$ with kernel

$$
\frac{\sin \pi \alpha}{2 \pi} \operatorname{sech}(x-y) / 2 \text {. }
$$

This is a finite Wiener-Hopf operator with symbol $\sin \pi \alpha \operatorname{sech} \pi \xi$ so we can use (2)). We have $G\left(1-\sin ^{2} \pi \alpha \operatorname{sech}^{2} \pi \xi\right)=e^{-\alpha^{2}}$. Since $E\left(\varphi_{r}\right)=\left(1-r^{2}\right)^{-\alpha^{2}}$ and $\varepsilon \sim(1-r) / 2$ we see that $E\left(\varphi_{r}\right) \varepsilon^{\alpha^{2}} \rightarrow 4^{-\alpha^{2}}$, which gives

$$
\lim _{r \rightarrow 1} E\left(\varphi_{r}\right) \operatorname{det}\left(I-A_{0}^{2}\right)=4^{-\alpha^{2}} E(1-\sin \pi \alpha \operatorname{sech} \pi \xi) E(1+\sin \pi \alpha \operatorname{sech} \pi \xi) .
$$

The limit of the factor

$$
\operatorname{det}\left(I-\left(I-A_{0}\right)^{-1} P\left(A-A_{0}\right)\right)
$$

in (6) is the determinant of what we get if we replace $P\left(A-A_{0}\right)$ by the operator $A_{1}$ on $L^{2}(0,1)$, by the established trace norm convergence. As for the factor

$$
\operatorname{det}\left(I-\left(I-A_{0}\right)^{-1} Q\left(A-A_{0}\right)\right) \text {, }
$$

notice that under the variable changes $x \rightarrow \varepsilon / x, y \rightarrow \varepsilon / y$ the operator $Q\left(A-A_{0}\right)$ on $L^{2}(\varepsilon, 1)$ becomes the one with kernel

$$
\chi_{[\sqrt{\varepsilon}, 1]}(x)\left[\left(\frac{1+x}{1-x}\right)^{\alpha}\left(\frac{x-\varepsilon}{x+\varepsilon}\right)^{n+\alpha}-1\right] \frac{1}{x+y} .
$$

And, in analogy with what went before, this converges in trace norm to the kernel $A_{2}(x, y)$ on $L^{2}(0,1)$. Under this substitution the kernel of $A_{0}$ is unchanged.

All this can be done with the other factors in (6). This establishes the lemma under our assumption that $\alpha$ is small enough. But the identity will hold in any connected $\alpha$-region containing 0 in which both sides are analytic, so where $\varphi_{\alpha, \alpha} \in L^{1}$ and where $I \pm A_{0}$ are invertible and $A_{1}$ and $A_{2}$ trace class. This holds for $|\Re \alpha|<1 / 2$.

Remark. The operators $\left.I \pm\left(I \pm A_{0}\right)^{-1} A_{1}\right)$, those involving $n$ in the statement of the lemma, are uniformly invertible for large $n$. It suffices to show uniform invertibility for $I \pm$ the operator with kernel the sine factor times

$$
\left(\frac{1-x}{1+x}\right)^{n+\alpha} \frac{1}{x+y}
$$


If we drop the $\alpha$ we make an error with operator norm $o(1)$, and then it suffices to prove the uniform invertibility of $I \pm$ the sine factor times

$$
\left(\frac{1-x}{1+x}\right)^{n / 2} \frac{1}{x+y}\left(\frac{1-y}{1+y}\right)^{n / 2}
$$

Since the spectrum of $1 /(x+y)$ is $[0, \pi]$ and the factors have absolute value at most 1 , the spectrum of the above also lies in $[0, \pi]$. Since it is self-adjoint the uniform invertibility follows whenever $1 / \sin \pi \alpha \notin[-1,1]$, and so when $|\Re \alpha|<1 / 2$.

Now let us go to Wiener-Hopf. To state the analogue of the Borodin-Okounkov identity here, let $\sigma$ be a symbol with regular logarithm. Write $\sigma=\sigma^{+} \sigma^{-}$where $\sigma^{+}$extends to be nonzero, bounded and analytic in the upper half-plane and $\sigma^{-}$in the lower and let $K_{R}$ be the operator on $L^{2}(0, \infty)$ with kernel

$$
K_{R}(x, y)=\int_{0}^{\infty}\left(\frac{\sigma^{-}}{\sigma^{+}}-1\right)_{R+x+z}\left(\frac{\sigma^{+}}{\sigma^{-}}-1\right)_{-R-z-y} d z
$$

(Here for notational convenience the subscripts denote Fourier transform.) Then for a symbol $\sigma$ with sufficiently regular logarithm and satisfying $\sigma-1 \in L^{1}$ we have

$$
\operatorname{det} W_{R}(\sigma)=G(\sigma)^{R} E(\sigma) \operatorname{det}\left(I-K_{R}\right) .
$$

The analogous formula for the regularized determinant is the following: for a symbol with sufficiently regular logarithm and satisfying $\sigma-1 \in L^{2}$,

$$
\operatorname{det}_{2} W_{R}(\sigma)=G_{2}(\sigma)^{R} E(\sigma) \operatorname{det}\left(I-K_{R}\right)
$$

The formula in the case that $\sigma-1 \in L^{1}$ follows from the results in [2]. The derivation of the formula when $\sigma-1 \in L^{2}$ is given in the Appendix.

We introduce a small parameter $\varepsilon$ and change $\sigma_{\alpha, \alpha}$ to the regular symbol

$$
\sigma_{\varepsilon}(\xi)=\left(\frac{\xi^{2}+\varepsilon^{2}}{\xi^{2}+1}\right)^{\alpha}
$$

As in the Toeplitz case we can compute the finite regularized Wiener-Hopf determinant of $\sigma_{\alpha, \alpha}$ for fixed $R$ by letting $\varepsilon$ tend to zero. To see that this is so observe that since $\sigma_{\varepsilon}(\xi)-1$ tends to $\sigma_{\alpha, \alpha}-1$ in $L^{2}$ the same is true of their Fourier transforms. Thus the corresponding kernels $k_{\varepsilon}(x-y)$ and $k(x-y)$ satisfy

$$
\int_{0}^{R} \int_{0}^{R}\left|k_{\varepsilon}(x-y)-k(x-y)\right|^{2} d x d y \leq R \int_{-\infty}^{\infty}\left|k_{\varepsilon}(x)-k(x)\right|^{2} d x
$$

which tends to zero. This shows that the regularized determinants are limits of ones with smooth symbols. This also holds for the $G_{2}\left(\sigma_{\alpha, \alpha}\right)$ term. (This discussion holds also for $\sigma_{\alpha, \beta}$ when $\alpha \neq \beta$. When $\alpha=\beta$ we have $\sigma_{\alpha, \alpha}-1 \in L^{1}$ and the above remarks hold for the ordinary determinant as well.) 
The constant $E\left(\sigma_{\varepsilon}\right)$ which arises in $(7)$ equals $\left((1+\varepsilon)^{2} / 4 \varepsilon\right)^{\alpha^{2}}$ and so $E\left(\sigma_{\varepsilon}\right) \varepsilon^{\alpha^{2}}$ has the same limit $4^{-\alpha^{2}}$ as in the Toeplitz case. If our finite Wiener-Hopf operator acts on $L^{2}(0, R)$ then the operator $K_{R}$ in (7) equals the square of the Hankel operator acting on $L^{2}(0, \infty)$ with kernel (in variables $s$ and $t$ )

$$
\begin{aligned}
& \frac{1}{2 \pi} \int_{-\infty}^{\infty}\left[\left(\frac{\xi+\varepsilon i}{\xi-\varepsilon i}\right)^{\alpha}\left(\frac{\xi-i}{\xi+i}\right)^{\alpha}-1\right] e^{i(R+s+t) \xi} d \xi \\
& =\frac{\sin \pi \alpha}{\pi} \int_{\varepsilon}^{1}\left(\frac{x+\varepsilon}{x-\varepsilon}\right)^{\alpha}\left(\frac{1-x}{1+x}\right)^{\alpha} e^{-(R+s+t) x} d x .
\end{aligned}
$$

Making a switch $U V \rightarrow V U$ as before changes this to the kernel

$$
\frac{\sin \pi \alpha}{\pi}\left(\frac{x+\varepsilon}{x-\varepsilon}\right)^{\alpha}\left(\frac{1-x}{1+x}\right)^{\alpha} e^{-R x} \frac{1}{x+y}
$$

on $L^{2}(\varepsilon, 1)$.

This is almost exactly the same as the kernel (5). The only difference is that the expression $((1-x) /(1+x))^{n+\alpha}$ there is replaced here by

$$
\left(\frac{1-x}{1+x}\right)^{\alpha} e^{-R x}
$$

The same argument given above for the Toeplitz case gives the following for the Wiener-Hopf case.

Lemma 5. Assume $|\Re \alpha|<1 / 2$. Let $\tilde{A}_{1}$ be the operator on $L^{2}(0,1)$ with kernel

$$
\tilde{A}_{1}(x, y)=\frac{\sin \pi \alpha}{\pi}\left[\left(\frac{1-x}{1+x}\right)^{\alpha} e^{-R x}-1\right] \frac{1}{x+y} .
$$

Then with $A_{0}$ and $A_{2}$ as in Lemma 5 we have

$$
\begin{gathered}
\operatorname{det}_{2} W_{R}\left(\sigma_{\alpha, \alpha}\right) / G_{2}\left(\sigma_{\alpha, \alpha}\right)^{R}=4^{-\alpha^{2}} E(1-\sin \pi \alpha \operatorname{sech} \pi \xi) E(1+\sin \pi \alpha \operatorname{sech} \pi \xi) \\
\times \operatorname{det}\left(I-\left(I-A_{0}\right)^{-1} \tilde{A}_{1}\right) \operatorname{det}\left(I+\left(I+A_{0}\right)^{-1} \tilde{A}_{1}\right) \operatorname{det}\left(I-\left(I-A_{0}\right)^{-1} A_{2}\right) \operatorname{det}\left(I+\left(I+A_{0}\right)^{-1} A_{2}\right) .
\end{gathered}
$$

We can now establish the theorem in the case $\alpha=\beta$. If we knew that $A_{1}-\tilde{A}_{1}=o_{1}(1)$ then this together with the uniform invertibility described in the remark following Lemma 4 would show that the determinants involving $A_{1}$ and $\tilde{A}_{1}$ are asymptotically equal. Also, because when $n$ is replaced by anything asymptotic to it the asymptotics of $T_{n}\left(\varphi_{\alpha, \alpha}\right)$ are the same, we may replace the condition on $R$ by the stronger one $R=2 n+O(1)$. We suppose first that $R=2 n$ exactly. If we apply Lemma 1 with $a=1 / 2$, say, we see that we have to show that

$$
\int_{0}^{1}\left|\left(\frac{1-x}{1+x}\right)^{n}-e^{-2 n x}\right|^{2} \frac{d x}{x^{3 / 2}}=o(1)
$$

as $n \rightarrow \infty$. The part of the integral where $x$ is bounded away from 0 is clearly $o(1)$. If $x$ is small, say $x<\delta$, we make a variable change $x \rightarrow x / 2 n$ and that part of the integral becomes a constant times

$$
n^{1 / 2} \int_{0}^{2 \delta n}\left|e^{-x+O\left(x^{2} / n\right)}-e^{-x}\right|^{2} \frac{d x}{x^{3 / 2}} .
$$


The integral over $x<\sqrt{n}$ is $O\left(n^{-2}\right)$ and the integral over $x>\sqrt{n}$ is $O\left(e^{-\sqrt{n}}\right)$ since the $O\left(x^{2} / n\right)$ term in the exponent is $<x / 2$ in the range of integration if $\delta$ is small enough. Thus $A_{1}-\tilde{A}_{1}=o_{1}(1)$ when $R=2 n$. The error incurred when our actual $R=2 n+O(1)$ is replaced by $2 n$ easily seen to be $o(1)$. This completes the proof of the theorem in the case $\alpha=\beta$.

To derive the corollary, observe that

$$
\begin{gathered}
\operatorname{det}_{2} T_{n}\left(\varphi_{\alpha, \alpha}\right)=\operatorname{det} T_{n}\left(\varphi_{\alpha, \alpha}\right) e^{-\operatorname{tr}\left(T_{n}\left(\varphi_{\alpha, \alpha}\right)-I\right)}, \\
\operatorname{det}_{2} W_{R}\left(\sigma_{\alpha, \alpha}\right)=\operatorname{det} W_{R}\left(\sigma_{\alpha, \alpha}\right) e^{-\operatorname{tr}\left(W_{n}\left(\sigma_{\alpha, \alpha}\right)-I\right)}, \\
\operatorname{tr}\left(T_{n}\left(\varphi_{\alpha, \alpha}\right)-I\right)=n\left(\left(\varphi_{\alpha, \alpha}\right)_{0}-1\right),
\end{gathered}
$$

and

$$
\operatorname{tr}\left(W_{R}\left(\sigma_{\alpha, \alpha}\right)-I\right)=R\left(\frac{1}{2 \pi} \int_{-\infty}^{\infty}\left(\sigma_{\alpha, \alpha}(\zeta)-1\right) d \zeta\right)
$$

(The geometric means for both $\sigma$ and $\varphi$ are one.)

Remark. To prove that the Toeplitz and Wiener-Hopf determinants are asymptotically equal it was clearly not necessary to have the identities given in Lemmas 4 and 5 . All that was needed were appropriate uniform invertibility and the fact that $A_{1}-\tilde{A}_{1}=o_{1}(1)$. We derived the identities because there was so little extra work involved.

\section{General $\alpha$ and $\beta$.}

Introducing a parameter $r$ now leads to the symbol $\varphi_{r}(z)=(1-r z)^{\alpha}\left(1-r z^{-1}\right)^{\beta}$. We have $E\left(\varphi_{r}\right)=\left(1-r^{2}\right)^{-\alpha \beta}$. Thus we are left with the contribution from $\operatorname{det}\left(I-K_{n}\right)$ just as in the previous case. Proceeding as we did before leads to the Fredholm determinant of the product of two different operators on $L^{2}(\varepsilon, 1)$. One has kernel

$$
2^{\beta-\alpha} \varepsilon^{\alpha-\beta} \frac{\sin \pi \beta}{\pi} \frac{(x+\varepsilon)^{\beta}}{(x-\varepsilon)^{\alpha}} \frac{(1-x)^{n+\alpha}}{(1+x)^{n+\beta}} \frac{1}{x+y}
$$

and the kernel of the other is obtained from this by interchanging $\alpha$ and $\beta$. For the product the factors $2^{\beta-\alpha} \varepsilon^{\alpha-\beta}$ and $2^{\alpha-\beta} \varepsilon^{\beta-\alpha}$ cancel, and so we can replace them both by 1 . The Fredholm determinant of the product equals the Fredholm determinant of the matrix kernel

$$
\left(\begin{array}{cc}
0 & \frac{\sin \pi \beta}{\pi} \frac{(x+\varepsilon)^{\beta}}{(x-\varepsilon)^{\alpha}} \frac{(1-x)^{n+\alpha}}{(1+x)^{n+\beta}} \frac{1}{x+y} \\
\frac{\sin \pi \alpha}{\pi} \frac{(x+\varepsilon)^{\alpha}}{(x-\varepsilon)^{\beta}} \frac{(1-x)^{n+\beta}}{(1+x)^{n+\alpha}} \frac{1}{x+y} & 0
\end{array}\right)
$$

We proceed as before, with $A_{0}$ having matrix kernel

$$
\left(\begin{array}{cc}
0 & \frac{\sin \pi \beta}{\pi} \frac{x^{\beta-\alpha}}{x+y} \\
\frac{\sin \pi \alpha}{\pi} \frac{x^{\alpha-\beta}}{x+y} & 0
\end{array}\right) .
$$

We assume at first that $\alpha$ and $\beta$ are small and purely imaginary. Then all $x$-factors which arise will be bounded. The analogue of (6) is here 


$$
\begin{gathered}
\operatorname{det}\left(I-K_{n}\right) \sim \operatorname{det}\left(I-A_{0}\right) \\
\times \operatorname{det}\left(I-\left(I-A_{0}\right)^{-1} P\left(A-A_{0}\right)\right) \operatorname{det}\left(I-\left(I-A_{0}\right)^{-1} Q\left(A-A_{0}\right)\right)
\end{gathered}
$$

as $r \rightarrow 1(\varepsilon \rightarrow 0)$. To see this, observe that $P\left(A-A_{0}\right)$ is $o_{1}(1)$ plus the operator with kernel

$$
\left(\begin{array}{cc}
0 & \frac{\sin \pi \beta}{\pi}\left[\frac{(1-x)^{n+\alpha}}{(1+x)^{n+\beta}}-1\right] \frac{x^{\beta-\alpha}}{x+y} \\
\frac{\sin \pi \alpha}{\pi}\left[\frac{(1-x)^{n+\beta}}{(1+x)^{n+\alpha}}-1\right] \frac{x^{\alpha-\beta}}{x+y} & 0
\end{array}\right) .
$$

This is true following the same argument as in Lemma 2 since the function $\frac{(x+\varepsilon)^{\alpha}}{(x-\varepsilon)^{\beta}}-x^{\alpha-\beta}$ (and also the one with $\alpha$ and $\beta$ interchanged) is $O(\sqrt{\varepsilon})$ for $x \in(\sqrt{\varepsilon}, 1)$. From this it follows that the analogues of Lemmas 2 and 3 hold and also (9).

Now we proceed to find the analogue of Lemma 4. The kernel $Q\left(A-A_{0}\right)$ is $o_{1}(1)$ plus the operator with kernel

$$
\chi_{(\varepsilon, \sqrt{\varepsilon})}(x)\left(\begin{array}{cc}
0 & \frac{\sin \pi \beta}{\pi}\left[\frac{(x+\varepsilon)^{\beta}}{(x-\varepsilon)^{\alpha}}-x^{\beta-\alpha}\right] \frac{1}{x+y} \\
\frac{\sin \pi \alpha}{\pi}\left[\frac{(x+\varepsilon)^{\alpha}}{(x-\varepsilon)^{\beta}}-x^{\alpha-\beta}\right] \frac{1}{x+y} & 0
\end{array}\right) .
$$

If we make the variable changes $x \rightarrow \varepsilon / x, y \rightarrow \varepsilon / y$ this becomes

$$
\chi_{(\sqrt{\varepsilon}, 1)}(x)\left(\begin{array}{cc}
0 & \varepsilon^{\beta-\alpha \frac{\sin \pi \beta}{\pi}}\left[\frac{(1+x)^{\beta}}{(1-x)^{\alpha}}-1\right] \frac{x^{\alpha-\beta}}{x+y} \\
\varepsilon^{\alpha-\beta \frac{\sin \pi \alpha}{\pi}\left[\frac{\left(1+x^{\alpha}\right.}{(1-x)^{\beta}}-1\right] \frac{x^{\beta-\alpha}}{x+y}} & 0
\end{array}\right),
$$

and the kernel of $A_{0}$ becomes

$$
\left(\begin{array}{cc}
0 & \varepsilon^{\beta-\alpha \frac{\sin \pi \beta}{\pi} \frac{x^{\alpha-\beta}}{x+y}} \\
\varepsilon^{\alpha-\beta \frac{\sin \pi \alpha}{\pi} \frac{x^{\beta-\alpha}}{x+y}} & 0
\end{array}\right) .
$$

Since determinants are unchanged if we multiply all kernels on the left by $\left(\begin{array}{cc}I & 0 \\ 0 & \varepsilon^{\beta-\alpha} I\end{array}\right)$ and on the right by $\left(\begin{array}{cc}I & 0 \\ 0 & \varepsilon^{\alpha-\beta} I\end{array}\right)$ we can remove these $\varepsilon$ factors from both kernels. Therefore for the limit of the second determinant in (9) the operators act on $L^{2}(0,1)$ and we replace the kernel of $A_{0}$ by

$$
\left(\begin{array}{cc}
0 & \frac{\sin \pi \beta}{\pi} \frac{x^{\alpha-\beta}}{x+y} \\
\frac{\sin \pi \alpha}{\pi} \frac{x^{\beta-\alpha}}{x+y} & 0
\end{array}\right)
$$

and that of $Q\left(A-A_{0}\right)$ by

$$
\left(\begin{array}{ccc}
0 & \frac{\sin \pi \beta}{\pi}\left[\frac{(1+x)^{\beta}}{(1-x)^{\alpha}}-1\right] \frac{x^{\alpha-\beta}}{x+y} \\
\frac{\sin \pi \alpha}{\pi}\left[\frac{\left(1+x^{\alpha}\right.}{(1-x)^{\beta}}-1\right] \frac{x^{\beta-\alpha}}{x+y} & 0
\end{array}\right) .
$$


Finally, for the limiting operators arising from the $P\left(A-A_{0}\right)$ term we multiply the matrix kernels on the left by $\left(\begin{array}{cc}x^{(\alpha-\beta) / 2} & 0 \\ 0 & x^{(\beta-\alpha) / 2}\end{array}\right)$ and on the right by $\left(\begin{array}{cc}y^{(\beta-\alpha) / 2} & 0 \\ 0 & y^{(\alpha-\beta) / 2}\end{array}\right)$. Thus the kernel of $A_{0}$ here becomes

$$
A_{0}(x, y)=\left(\begin{array}{cc}
0 & \frac{\sin \pi \beta}{\pi} \frac{(x / y)^{(\beta-\alpha) / 2}}{x+y} \\
\frac{\sin \pi \alpha}{\pi} \frac{(x / y)^{(\alpha-\beta) / 2}}{x+y} & 0
\end{array}\right)
$$

and the kernel coming from $P\left(A-A_{0}\right)$ becomes

$$
A_{1}(x, y)=\left(\begin{array}{cc}
0 & \frac{\sin \pi \beta}{\pi}\left[\frac{(1-x)^{n+\alpha}}{(1+x)^{n+\beta}}-1\right] \frac{(x / y)^{(\beta-\alpha) / 2}}{x+y} \\
\frac{\sin \pi \alpha}{\pi}\left[\frac{(1-x)^{n+\beta}}{(1+x)^{n+\alpha}}-1\right] \frac{(x / y)^{(\alpha-\beta) / 2}}{x+y} & 0
\end{array}\right) .
$$

Similarly, for the limiting operators arising from the $Q\left(A-A_{0}\right)$ term we multiply the matrix kernels on the left by $\left(\begin{array}{cc}x^{(\beta-\alpha) / 2} & 0 \\ 0 & x^{(\alpha-\beta) / 2}\end{array}\right)$ and on the right by $\left(\begin{array}{cc}y^{(\alpha-\beta) / 2} & 0 \\ 0 & y^{(\beta-\alpha) / 2}\end{array}\right)$. The kernel of $A_{0}$ here becomes

$$
A_{0}(x, y)=\left(\begin{array}{cc}
0 & \frac{\sin \pi \beta}{\pi} \frac{(x / y)^{(\alpha-\beta) / 2}}{x+y} \\
\frac{\sin \pi \alpha}{\pi} \frac{(x / y)^{(\beta-\alpha) / 2}}{x+y} & 0
\end{array}\right)
$$

and the kernel coming from $Q\left(A-A_{0}\right)$ becomes

$$
A_{2}(x, y)=\left(\begin{array}{cc}
0 & \frac{\sin \pi \beta}{\pi}\left[\frac{(1+x)^{\beta}}{(1-x)^{\alpha}}-1\right] \frac{(x / y)^{(\alpha-\beta) / 2}}{x+y} \\
\frac{\sin \pi \alpha}{\pi}\left[\frac{\left(1+x^{\alpha}\right.}{(1-x)^{\beta}}-1\right] \frac{(x / y)^{(\beta-\alpha) / 2}}{x+y} & 0
\end{array}\right) .
$$

None of these operations change the determinants.

For the asymptotics of $\operatorname{det}\left(I-A_{0}\right)$ in (9) both versions (10) and (12) give the same determinant. If we make the variable changes $x \rightarrow e^{-x}, y \rightarrow e^{-y}$ then (10) becomes

$$
\left(\begin{array}{cc}
0 & \frac{\sin \pi \beta}{2 \pi} \frac{e^{(\beta-\alpha)(x-y) / 2}}{\cosh (x-y) / 2} \\
\frac{\sin \pi \alpha}{2 \pi} \frac{e^{(\alpha-\beta)(x-y) / 2}}{\cosh (x-y) / 2} & 0
\end{array}\right)
$$

on $L^{2}(0, \infty)$. This is the kernel of a Wiener-Hopf operator with matrix symbol.

The matrix version of (2) is well known. The constant $G(\sigma)$ is defined now by using the logarithm of the determinant of the symbol and $E(\sigma)$ is replaced by $\operatorname{det} W(\sigma) W\left(\sigma^{-1}\right)$. The symbol of $I$ minus the operator with kernel (14) is

$$
\tau(\xi)=\left(\begin{array}{cc}
1 & -\frac{\sin \pi \beta}{\cosh \pi(\xi+i(\alpha-\beta) / 2)} \\
-\frac{\sin \pi \alpha}{\cosh \pi(\xi-i(\alpha-\beta) 2)} & 1
\end{array}\right),
$$


whose determinant equals

$$
1-\frac{\sin \pi \alpha \sin \pi \beta}{\cosh ^{2} \pi \xi-\sin ^{2} \pi(\alpha-\beta) / 2}
$$

Using the matrix version of (2) we find that the $G$ factor times the Toeplitz $E\left(\varphi_{r}\right)$ has limit $4^{-\alpha \beta}$ while the $E$ factor equals $\operatorname{det} W(\tau) W\left(\tau^{-1}\right)$.

If we set $C(\alpha, \beta)=4^{-\alpha \beta} \operatorname{det} W(\tau) W\left(\tau^{-1}\right)$ we see that we have arrived at the following point.

Lemma 6. If $\alpha$ and $\beta$ are sufficiently small and purely imaginary then

$$
\operatorname{det}_{2} T_{n}\left(\varphi_{\alpha, \beta}\right) / G_{2}\left(\varphi_{\alpha, \beta}\right)^{n}=C(\alpha, \beta) \operatorname{det}\left(I-\left(I-A_{0}\right)^{-1} A_{1}\right) \operatorname{det}\left(I-\left(I-A_{0}\right)^{-1} A_{2}\right) .
$$

In the first determinant on the right the kernels of $A_{0}$ and $A_{1}$ are given by (10) and (11) and in the second determinant the kernels of $A_{0}$ and $A_{2}$ are given by (12) and (13).

This can be extended to any connected $(\alpha, \beta)$ region where $A_{1}$ and $A_{2}$ are trace class and the $I-A_{0}$ (both versions) are invertible. Requirements just for boundedness of the operators are $\Re \alpha, \Re \beta<1 / 2$ and $|\Re(\alpha-\beta)|<1$. An application of Lemma 2 shows that these suffice also for the entries of $A_{1}$ and $A_{2}$ to be trace class. (Under this condition an $a$ can be found in each case such that the integrals that arise are finite.) As for the invertibility of $I-A_{0}$, a little trigonometry shows that (15) is nonzero if $\cos \pi(\alpha+\beta) / 2 \notin(-\infty,-1]$, and this holds if $|\Re(\alpha+\beta)|<1$. Since this is a connected set and the index of the determinant is zero for $\alpha$ and $\beta$ small it must be zero for all these $(\alpha, \beta)$. Hence under this condition $I-A_{0}$ is Fredholm of index zero. But we do not know that it is invertible, so here is what we do. We know that $W(\tau)$ is invertible if $W(\tau) W\left(\tau^{-1}\right)$ is. This operator is of the form $I$ plus trace class and its determinant is an analytic function of $\alpha$ and $\beta$ for $|\Re(\alpha \pm \beta)|<1$. So we assume temporarily that $(\alpha, \beta)$ is not in the zero set of this determinant. (Notice that the determinant is nonzero for $\alpha$ and $\beta$ are small enough.) The same applies to the other version of $A_{0}$. Recall that we still have the extra requirement that $\Re \alpha, \Re \beta<1 / 2$.

Once we have invertibility of both versions of $I-A_{0}$ we can proceed as before to the Wiener-Hopf analogue. Now the only change is that the expressions involving $1 \pm x$ appearing in (11) are replaced by $(1-x)^{\alpha}(1+x)^{-\beta} e^{-R x}$ and $(1-x)^{\beta}(1+x)^{-\alpha} e^{-R x}$ so we must add the assumption that $\Re \alpha, \Re \beta>-1 / 2$. Other than this everything is as before until when we come to uniform invertibility. After replacing those expressions by $e^{-2 n x}$ (the error in doing this being an operator with norm $o(1)$ ), the problem becomes that of uniform invertibility of $I$ minus

$$
\left(\begin{array}{cc}
0 & \frac{\sin \pi \beta}{\pi} e^{-2 n x} \frac{(x / y)^{(\beta-\alpha) / 2}}{x+y} \\
\frac{\sin \pi \alpha}{\pi} e^{-2 n x} \frac{(x / y)^{(\alpha-\beta) / 2}}{x+y} & 0
\end{array}\right)
$$

on $L^{2}(0,1)$. We see upon making the substitutions $x \rightarrow x / 2 n, y \rightarrow y / 2 n$ that this is 
equivalent to the uniform invertibility of $I$ minus

$$
\left(\begin{array}{cc}
0 & \frac{\sin \pi \beta}{\pi} e^{-x} \frac{(x / y)^{(\beta-\alpha) / 2}}{x+y} \\
\frac{\sin \pi \alpha}{\pi} e^{-x} \frac{(x / y)^{(\alpha-\beta) / 2}}{x+y} & 0
\end{array}\right)
$$

on $L^{2}(0,2 n)$. This is completely equivalent to the invertibility of $I$ minus the operator with the same kernel on $L^{2}(0, \infty)$, and this in turn is equivalent to the invertibility of $I$ minus the operator $L$ with kernel

$$
L(x, y)=\left(\begin{array}{cc}
0 & \frac{\sin \pi \beta}{\pi} e^{-x / 2} \frac{(x / y)^{(\beta-\alpha) / 2}}{x+y} e^{-y / 2} \\
\frac{\sin \pi \alpha}{\pi} e^{-x / 2} \frac{(x / y)^{(\alpha-\beta) / 2}}{x+y} e^{-y / 2} & 0
\end{array}\right) .
$$

Lemma 7. The operator $I-L$ is invertible when $|\Re(\alpha \pm \beta)|<1$ and $(\alpha, \beta)$ does not lie in the zero set of some analytic function which is nonzero for sufficiently small $\alpha$ and $\beta$.

Proof. Let $P$ denote multiplication by $\chi_{[0,1]}$ and think of the kernel $A_{0}$ given by (10) as acting on $L^{2}(0, \infty)$. We know that $I-P A_{0} P$ is invertible except for $(\alpha, \beta)$ in the zero set of some analytic function which is nonzero for $\alpha$ and $\beta$ small. Now we can write

$$
I-L=I-P A_{0} P-P\left(L-A_{0}\right) P-P L(I-P)-(I-P) L P
$$

and several applications of Lemma 2 show that the operators $P\left(L-A_{0}\right) P, P L(I-P)$, and $(I-P) L P$ are all trace class. Hence whenever $I-P A_{0} P$ is invertible the invertibility of $I-L$ is equivalent to the invertibility of $I-\left(I-P A_{0} P\right)^{-1}\left(P\left(L-A_{0}\right) P+P L(I-P)+(I-P) L P\right)$ which in turn is equivalent to the nonvanishing of its determinant. Since this is analytic in $\alpha$ and $\beta$ and nonzero for small $\alpha$ and $\beta$ the assertion follows.

Having established the necessary invertibility and uniform invertibility the asymptotics stated in the theorem follow, as in the case $\alpha=\beta$. But recall that we still have two conditions beyond the hypothesis $|\Re(\alpha \pm \beta)|<1$ of the theorem:

(i) $(\alpha, \beta)$ does not lie in the zero set of some analytic function $F(\alpha, \beta)$ which is nonzero for sufficiently small $\alpha$ and $\beta$.

(ii) $|\Re \alpha|,|\Re \beta|<1 / 2$.

To remove requirement (i) we use the analyticity of the regularized determinants and geometric means, the latter also being nonzero. Suppose $\alpha$ and $\beta$ satisfy (ii). The set $S$ of $\alpha$ for which $F(\alpha, \beta)$ is identically 0 in $\beta$ is discrete. Assume $\alpha \notin S$. Then $\{\beta: F(\alpha, \beta)=0\}$ is discrete. Choose any $\beta$ with $|\Re \beta|<1 / 2$. There is a little circle $\Gamma$ with center $\beta$ such that $F\left(\alpha, \beta^{\prime}\right) \neq 0$ for all $\beta^{\prime} \in \Gamma$. We know that

$$
\frac{\operatorname{det}_{2} W_{R}\left(\sigma_{\alpha, \beta^{\prime}}\right) / G_{2}\left(\sigma_{\alpha, \beta^{\prime}}\right)^{R}}{\operatorname{det}_{2} T_{n}\left(\varphi_{\alpha, \beta^{\prime}}\right) / G_{2}\left(\varphi_{\alpha, \beta^{\prime}}\right)^{n}} \rightarrow 1
$$


for all $\beta^{\prime} \in \Gamma$, and the denominator is also nonzero for $\beta^{\prime}$ inside $\Gamma$ by the known asymptotics of $\operatorname{det} T_{n}\left(\varphi_{\alpha, \beta^{\prime}}\right)$. The limit holds uniformly for all $\beta^{\prime} \in \Gamma$. Therefore the limit holds for $\beta^{\prime}=\beta$ as well. So the statement of the theorem holds for all $\alpha$ satisfying $|\Re \alpha|<1 / 2$ except for those lying in $S$. But now we can repeat the previous argument to show that it holds for $\alpha \in S$ as well.

To remove requirement (ii) we do now what we did not do earlier only because it would have made the formulas yet more complicated. We had these requirements because of the factors involving powers of $1-x$ in (13) and the Wiener-Hopf analogue of (11). The exponents had to have real part greater than $-1 / 2$ for the $x$-factors to belong to $L^{2}$. What we could have done is multiply (12) and (13) on the left by $\left(\begin{array}{cc}(1-x)^{\alpha / 2} & 0 \\ 0 & (1-x)^{\beta / 2}\end{array}\right)$ and on the right by $\left(\begin{array}{cc}(1-y)^{-\alpha / 2} & 0 \\ 0 & (1-y)^{-\beta / 2}\end{array}\right)$, and multiply the Wiener-Hopf analogues of (10) and (11) on the left by $\left(\begin{array}{cc}(1-x)^{-\alpha / 2} & 0 \\ 0 & (1-x)^{-\beta / 2}\end{array}\right)$ and on the right by $\left(\begin{array}{cc}(1-y)^{\alpha / 2} & 0 \\ 0 & (1-y)^{\beta / 2}\end{array}\right)$. These would not have affected the determinants but the $x$-factors (and now also the $y$-factors) belong to $L^{2}$ under the weaker conditions $|\Re \alpha|,|\Re \beta|<1$. These hold under the hypothesis of the theorem and so are not extra conditions. The succeeding argument holds with only minor changes with these replacements.

\section{Appendix.}

The continuous analogue of the Borodin-Okounkov identity for generalized determinants is given by the formula

$$
\operatorname{det}_{2} W_{R}(\sigma)=G_{2}(\sigma)^{R} E(\sigma) \operatorname{det}\left(I-K_{R}\right)
$$

where the terms $G_{2}, E$ and $K_{R}$ were defined in Section II. The identity is valid if $\sigma-1 \in L^{2}$, if the Fourier transform $k$ of $\sigma-1$ is in $L^{1}$ and satisfies $\int_{-\infty}^{\infty}|x||k(x)|^{2} d x<\infty$, and finally if $\sigma$ is nonzero and has index zero. As we shall see, the last two assumptions imply that $\sigma=\sigma^{+} \sigma^{-}$where $\sigma^{+}$extends to be nonzero, bounded and analytic in the upper half-plane and $\sigma^{-}$in the lower. All factors tend to one at $\pm \infty$.

That (16) is true if $\sigma-1 \in L^{1} \cap L^{2}$ follows from the corresponding identity for the ordinary determinant proved in [2] and the formula

$$
\operatorname{det}_{2} A=\operatorname{det} A e^{-\operatorname{tr}(A-I)} \text {. }
$$

To prove (16) under the more general condition $\sigma \in L^{2}$ we shall approximate $\sigma-1$ by a sequence of functions in $\sigma_{n} \in L^{1} \cap L^{2}$, apply the identity to each $\sigma_{n}$ and then take a limit. In order to guarantee convergence of the terms appearing in the identity, we use a Banach algebra approach. We define the algebra $\mathcal{K}$ as the set of all bounded functions $\psi$ whose distributional Fourier transform on $\mathbf{R}-\{0\}$ is equal to a function $\hat{\psi}$ satisfying

$$
\|\psi\|_{\mathcal{K}}:=\int_{-\infty}^{\infty}|\hat{\psi}(\zeta)| d \zeta+\left(\int_{-\infty}^{\infty}|x||\hat{\psi}(\zeta)|^{2} d \zeta\right)^{1 / 2}<\infty
$$

This is a subalgebra of $L^{\infty}$ and it is clear that the function $\sigma$ is contained in the algebra. In [14] it was proved that if $\psi$ is in $\mathcal{K} \cap L^{2}$ then there is a sequence of functions $\psi_{n}$ with 
compact support converging to $\psi$ in both the norm of $L^{2}$ and $\mathcal{K}$. This was actually proved for a slightly larger Banach algebra, but the proof is the same here. If we apply this result to the function $\sigma-1$ we find a sequence of functions $\sigma_{n}-1$ for which (16) holds.

It remains to show that each of the various terms has the proper limit. Since $\sigma_{n}-1$ converges in $L^{2}$ to $\sigma-1$ the terms $\operatorname{det}_{2} W_{R}\left(\sigma_{n}\right)$ and $G_{2}\left(\sigma_{n}\right)^{R}$ converge to $\operatorname{det}_{2} W_{R}(\sigma)$ and $G_{2}(\sigma)^{R}$, respectively. The factor $E\left(\sigma_{n}\right)$ is equal to the $\operatorname{det} W\left(\sigma_{n}\right) W\left(\sigma_{n}^{-1}\right)=I-H\left(\sigma_{n}\right) H\left(\tilde{\sigma}_{n}^{-1}\right)$, where $H(\sigma)$ is a Hankel operator with kernel $\hat{\sigma}(x+y)$ and $H(\tilde{\sigma})$ is a Hankel operator with kernel $\hat{\sigma}(-x-y)$ Now since $\sigma_{n}$ converge in $\mathcal{K}$ to $\sigma$ it follows that the analogous statement is true for $\sigma^{-1}$ and thus the corresponding Hankel operators converge in the Hilbert Schmidt norm. This follows immediately from the definition of the norm on $\mathcal{K}$. Finally, convergence in $\mathcal{K}$ holds for the sequences $\sigma_{n}^{+}$and $\sigma_{n}^{-}$and their quotients, because the well-known projections used in their definitions are continuous in $\mathcal{K}$. This implies the convergence of the term $\operatorname{det}\left(I-K_{R}\left(\sigma_{n}\right)\right)$ to the corresponding $\operatorname{det}\left(I-K_{R}\right)$ and completes the proof of the identity.

\section{Acknowledgments}

The first author was supported by National Science Foundation grant DMS-9970879 and the second author by grant DMS-9732687.

\section{References}

[1] E. L. Basor, A localization theorem for Toeplitz determinants, Indiana Univ. Math. J. 28:6 (1979) 975-983.

[2] E. L. Basor and Y. Chen, A note on Wiener-Hopf determinants and the BorodinOkounkov identity, to appear in Int. Eqns. Operator Th.

[3] E. L. Basor and J. W. Helton, A new proof of the Szegö limit theorem and new results for Toeplitz operators with discontinuous symbol, J. Operator Th. 3 (1980) 23-29.

[4] E. L. Basor and H. Widom, Toeplitz and Wiener-Hopf determinants with piecewise continuous symbols, J. Funct. Anal. 50 (1983) 387-413.

[5] A. Borodin and A. Okounkov, A Fredholm determinant formula for Toeplitz determinants. Int. Eqns. Operator Th. 37 (2000) 386-396.

[6] A. Böttcher, Toeplitz determinants with piecewise continuous generating function, Z. Anal. Anw. 1:2 (1982) 23-39.

[7] A. Böttcher and B. Silbermann, Toeplitz determinants generated by symbols with one singularity of Fisher-Hartwig type, Wiss. Z. Tech. Hochsch. Karl-marx-Stadt 26:2 (1984) 186-188.

[8] A. Böttcher and B. Silbermann, Analysis of Toeplitz Operators, Akademie-Verlag Berlin, 1990. 
[9] A. Böttcher, B. Silbermann, and H. Widom A continuous analogue of the FisherHartwig formula for piecewise continuous symbols, J. Func. Anal. 122 (1994) 222-246.

[10] T. Ehrhardt, B. Silbermann, Toeplitz determinants with one Fisher-hartwig singularity, J. Func. Anal. bf 148 (1997), 229-256.

[11] T. Ehrhardt, A status report on the asymptotic behavior of Toeplitz determinants with Fisher-Hartwig singularities, Operator Th: Advances and App. 124 (2001), 217-241.

[12] M. E. Fisher and R. E. Hartwig, Toeplitz determinants: some applications, theorems, and conjectures, Adv. Chem. Phys. 15 (1968) 333-353.

[13] I. C. Gohberg and M. G. Krein, Introduction to the Theory of Linear Nonselfadjoint Operators, Transl. Math. Monographs 18, Amer. Math. Soc., Providence, 1969.

[14] H. Widom, On Wiener-Hopf determinants, Operator Th.: Adv. Appl. 41 (1989) 519543. 